Received 21 January 2019 Accepted 26 March 2019 Published 31 March 2019

Production and Hosting by Knowledge E

(c) Suad $\mathrm{HH}$ et al. This article is distributed under the terms of the Creative Commons Attribution License, which permits unrestricted use and redistribution provided that the original author and source are credited.

Editor-in-Chief:

Prof. Mohammad A. M. Ibnouf

\section{Treatment of Extensive Plaque Psoriasis and Psoriatic Arthropathy Using Methotrexate in a Patient with High Serum Alkaline Phosphatase}

\author{
Suad HH${ }^{1}$, Muna Mohammed El Amin ${ }^{2}$, Gad Allah Modawe ${ }^{3}$, and \\ Osman Elbasheer ${ }^{4}$ \\ ${ }^{1}$ Department of Dermatology, Omdurman Islamic University, Omdurman, Sudan \\ ${ }^{2}$ Department of Community Medicine, Omdurman Islamic university, Omdurman, Sudan \\ ${ }^{3}$ Department of Biochemistry, Omdurman Islamic University, Omdurman, Sudan \\ ${ }^{4}$ Khartoum Dermatology Teaching Hospital, Khartoum State, Sudan
}

\section{Abstract}

Background: Psoriasis is an autoimmune inflammatory, chronic, and relapsing disease of the skin, which can also present with severe joint inflammation and deformity. There are many treatment options, but the severe forms of psoriasis are treated successfully with cytotoxic and biologic therapies. Methotrexate being cheap and available in Sudan is considered a good option for patients with severe and extensive forms of psoriasis. One of the main drawbacks is hepatotoxicity. The aim of this report is to share an experience of treating the patient with oral methotrexate in the presence of a relative contraindication to this therapy.

Method: We present a 60-year-old female with extensive skin psoriasis and psoriatic arthropathy. Investigations showed high serum alkaline phosphatase and low serum albumen. Other liver enzymes, $\mathrm{CBC}$, ultrasound abdomen were all unremarkable. Patient was admitted and a weekly dose of methotrexate was given in addition to topical preparation and supportive treatment; three months later, patient improved and was moved to topical therapy. A check of serum alkaline phosphatase and albumen every week was done.

Results: The patient was successfully and safely treated with methotrexate. Serum alkaline phosphatase was normal within the second month treatment, and serum albumen was normal after only one month of treatment.

Conclusion: High serum alkaline phosphatase is considered a relative contraindication to the use of methotrexate, high level can be found in bone diseases. Serum albumen can be low in dermatologic diseases with excessive shedding of scales and can be corrected with protein formulas and high protein diets.

Recommendations: Methotrexate is recommended for the treatment of severe morbid forms of psoriasis. The biologic therapy for severe forms of psoriasis is now available and can be a good alternative for the treatment of severe forms of psoriasis in good centers and for patients who can afford and in patients with absolute contraindications to methotrexate therapy. 


\section{Introduction}

Psoriasis is a particular autoimmune systemic inflammatory disease of great interest. It has a chronic, relapsing, and remitting course that has devastating impact on a person's health-related quality of life (HRQL) [1].

Psoriasis is a common health problem with an estimated worldwide prevalence of $2-3 \%$, with great regional and ethnic variations. Caucasians predominate [2].

Few studies have reported on the incidence of psoriasis in the Middle East, including two small single-center studies conducted in 2004 that found the incidence of psoriasis to be 3.4\%, in the south-western and eastern Saudi Arabia (3).

Similar to all autoimmune diseases, the pathophysiology of psoriasis is so complicated, not yet fully understood, and expresses a mixture of genetic, environmental and behavioral influences. While, clinically, the signs and symptoms appear to be caused by hyper proliferation and inflammation of epidermal cells. In psoriasis, an immunologic response involving T-lymphocytes and cytokines alters the epidermal skin cell cycle to result in the formation of the psoriatic plaques, the whole mark of the disease [4].

Since the mid-1950s, methotrexate has become the gold standard by which other systemic psoriasis medications are measured (5).

In 1972, the Food and Drug Administration (FDA) approved methotrexate for the treatment of psoriasis and provided a formal basis for its use. Guidelines are published since this approval to standardize the use of methotrexate. These guidelines addressed indications, contraindications, pretreatment evaluation, monitoring of therapy, dosages, adverse reactions, and overdose. The original guidelines endorsed liver biopsy as the gold standard for detecting liver damage and introduced a histological classification standard for grading liver biopsy specimens. Since their original publication in 1972, the guidelines have continued to evolve over the years and have undergone four revisions $[6,7]$.

The latest set was published in 1998 where liver biopsies were replaced by blood tests for liver fibrosis, serum aminoterminal propeptide of type III procollagen (PIIIPN), based on results of small studies [8].

Methotrexate is a relatively safe medication as a serious damage to the liver undisputedly occurs in less than $1 \%$ or even less than $0.1 \%$ of the patients. The burden for patients might not outweigh the potential damage [9].

Efforts to treat severe psoriasis continues to increase; in 1974, a survey revealed that $52 \%$ of dermatologists used methotrexate to treat psoriasis, this increased in 1987 when another survey showed that the number of dermatologists who used methotrexate had 
increased slightly to $58 \%$. In 2001, millions of prescriptions were written for methotrexate, of which approximately $11 \%$ were for psoriasis (9).

Administered either as monotherapy or in combination with the other schemes, methotrexate exerts its effects by acting as both immunomodulatory and anti-metabolite agent due to the downregulation of T-cell-mediated pro-inflammatory markers and inhibition growth of keratinocytes and decrease in the endothelial expression of ICAM-1 and E-selectin (10).

Clinical data from trials suggest that $24 \%$ and $100 \%$ of psoriasis patients achieved PASI-75 at week 12 at MTX dosage of 7.5 and $15 \mathrm{mg}$, respectively. However, we found that $73 \%$ and $40 \%$ of patients reached PASI-75 at week 12 in group B and A, respectively (11).

During 1985, Lanes et al. had followed 30 patients with psoriasis and other nonmalignant diseases with liver biopsies done before treatment with low-dose methotrexate, $15 \mathrm{mg} /$ week, and then at one- to two-year intervals as long as they continued the methotrexate. All patients were symptomatically improved on this regimen. The 15 patients who had normal liver biopsies at the start of the study had normal biopsies after methotrexate, 15 others had minor hepatic histological abnormalities before treatment, 11 had fatty infiltration, 10 showed no significant change after treatment, while 1 had increased fat and portal fibrosis on a fourth liver biopsy done seven years after MTX was begun. This last patient, a former alcohol abuser, continued methotrexate and showed no further worsening at 8 years. The remaining four had portal fibrosis before treatment. One patient had less fibrosis after methotrexate, two patients slightly more fibrosis, and one a marked increase in portal fibrosis. No patient developed cirrhosis or clinical liver disease. These results suggest that in the absence of alcohol consumption, low-dose weekly methotrexate treatment rarely causes clinically significant liver damage.

Similar results for hepatotoxicity associated with low-dose, long-term methotrexate treatment of rheumatoid arthritis was shown by Szanto et al. in 1987.

Relative contraindication for methotrexate therapy include kidney failure, gastric ulcer, hypoalbuminemia, elevated liver enzymes, active infectious diseases, treatment with immunosuppressant, interactions with other drugs, hyperlipidemia, folic-acid deficiency, alcohol abuse, obesity, diabetes, active or past history of hepatitis, lack of patient compliance, and old age. While there are some other conditions that are considered absolute contraindications for treatment with methotrexate (12).

Case report: A 60-year old female, married and residing in Gezeira state has a known case of chronic plaque psoriasis since 20 years, with remissions and relapses, emollients and some preparations being used according to dermatologist prescription. 
In the last presentation, the patient had an extensive involvement of her skin affecting the extensors of limbs, face, trunk more marked over the lower parts and scalp. The condition was associated with severe bilateral hip joint pain and finger deformities, so severe that the patient had to use a wheel chair.

Pt was otherwise normal, no complains related to other systems, no abdominal pain, no chest pain, no palpitations or headache. No past history of schistosomiasis, hematuria, malèna, jaundice, or liver disease. No previous hospital admission. Pt not known diabetic or hypertensive. No family history of psoriasis or other skin disease. No history of alcohol consumption. Not on chronic medication, apart from the preparations for psoriasis. Patient is a house wife; her husband is an employee in a company with a low income, she is postmenopausal, with two daughters and two sons.

O I E: Patient was uncomfortable, pushed on a wheel chair. Not pale or jaundiced. Normal pulse and BP. No palpable lymph nodes. Lower limbs: pitting edema present bilaterally.

Examination of the chest and heart revealed no abnormality.

Abdomen showed no tenderness, no organomegaly. Liver was of normal size.

Skin: Lesions were located over the extensors of both upper and lower limbs, face and trunk involvement was more extensive on the lower half with no specific pattern of distribution in the form of well-demarcated papules and plaques covered with easily detached white silvery scales. The skin between the lesions is erythematous and tender.

Scalp: All covered with easily detached scales with well-demarcated psoriatic corona.

Palms and soles show hyperkeratosis. Mucous membranes were normal.

Joints: Painful interphalangeal joints with finger deformities. Bilateral painful tender hips.

Investigations: Urine and stool analysis showed no abnormality. CBC showed slightly raised WBC. RBCs, $\mathrm{Hb}$, and platelets were all normal.

Liver function tests: Slight increase of alkaline phosphatase and Low serum albumen, other liver enzymes were all normal.

Renal function tests and electrolytes were normal.

Rheumatoid factor -ve.

US abdomen: No organomegaly, liver is of normal size and texture, and no evidence of chronic liver disease.

X-Ray hips and X-Ray hands: inflammatory changes consistent with psoriatic arthritis. 
CXR: clear.

ECG: Normal.

\section{Plan of Management}

Admission, treatment with General measures included emollients, protein formulas, high protein diet, tonics, and systemic antibiotics. Specific measures included methotrexate tabs $15 \mathrm{mg} /$ week divided in three doses 12 hours apart with $5 \mathrm{mg}$ folic acid tabs, skin preparation included 5\% salicylic acid and $4 \%$ coal tar in $100 \mathrm{~g}$ Vaseline once a day. Coal tar shampoo for the scalp every other day.

Follow-up included daily assessment of the clinical state, mainly for the regression of psoriatic plaques, erythema, scales, and lower limb edema. Eye examination for jaundice is important, hip and finger examination for normal non-restricted joint movement should all be performed. Follow-up by investigations included weekly measurement of liver function tests with enzymes and CBC.

\section{Outcome}

After the second week, the condition improved in terms of edema, joint pain, and skin condition. In the third and fourth weeks, much improvement was seen and the patient could stand with support. The second month, the patient could walk with support, and so static improvement was noticed. At the end of the third month, the patient was discharged in good condition and could walk unsupported with marked clinical improvement of the skin condition on topical treatment with coal tar in Vaseline and divobet (divonex + betamethasone) cream. Follow-up by investigations showed weekly reduction of alkaline phosphatase level and was normal by the fifth week. Hemoglobin level was low in the third month for which ferrous sulphate was prescribed. Serum albumin was corrected by diet and protein formula, and it was normal after one month.

On discharge, liver function test was normal including alkaline phosphatase. Patient was discharged in good condition with respect to the clinical and laboratory status.

\section{Discussion}

Psoriasis is an autoimmune chronic inflammatory skin disease with remissions and relapses; it can affect joints causing psoriatic arthritis and can be so severe leading to erythroderma. 
The treatment of the localized forms can usually improve with topical agents, but severe forms like extensive plaque psoriasis, pustular psoriasis, psoriatic arthritis, and erythrodermic psoriasis need systemic treatment as well. Psoriatic arthritis occurs in $30 \%$ of patients. It can result in permanent joint damage, methotrexate is the most commonly prescribed medication together with NSAIDS.

Methotrexate is a relatively safe cytotoxic antimetabolite drug that has been approved by the FDA for the treatment of severe variants of psoriasis since 1972 [6, 7]. Serious damage due to methotrexate occurs in less than $1 \%$ or even less than $0.1 \%$ of patients [9]. It is effective in the treatment of severe forms of psoriasis, it acts as immunomodulatory and antimetabolite due to the down regulation of T-cell-mediated pro-inflammatory markers (10).

Studies done showed that in the absence of alcohol consumption, a weekly low dose of methotrexate rarely causes clinically significant liver damage. Guidelines for the use of methotrexate had been studied and clearly stated [6, 7].

The treatment of the reported case was considered challenging because of the need to a systemic medication, the options are methotrexate, cyclosporine and biologic therapy; the last two are very expensive in Sudan and the patient couldn't afford it; the former is cheap but the patient has an elevated alkaline phosphatase and low serum albumin which are considered relative contraindications for its use (12). In one study, portal fibrosis was encountered after seven years of use of methotrexate in a patient with psoriasis. Follow-up of liver damage was done through biopsies which were then replaced by the blood tests in 1998 by FDA (8).

Alkaline phosphatase is found in various tissues in the body, highest concentrations being found in the liver and bones; elevated levels can indicate tissue damage mainly bone or liver.

Hypo albuminemia can be found in patients with scaly skin diseases where excessive shedding of scales takes place.

The American Academy of Dermatology suggests the following investigations before starting treatment with methotrexate: $\mathrm{CBC}, \mathrm{S}$. Creatinine, blood urea nitrogen, and liver function tests with enzymes. Depending on all of the aforementioned facts, a decision for managing the patient with methotrexate was made. The plan was: Admission, base line investigations, weekly oral methotrexate, topical antipsoriasis treatment, systemic antibiotics for UTI, supportive therapy and tonics, clinical follow-up, and through investigations such as liver function tests and CBC. 
Baseline investigations done were normal apart from minor UTI, elevated serum alkaline phosphatase, low serum albumin, inflammatory changes of hand and hip joints indicating psoriatic arthritis.

The patient was well managed with systemic therapy using weekly doses of methotrexate in addition to topical therapy using salicylic acid and coal tar preparations.

The treatment of this patient was really challenging. Methotrexate is a cheap medication but it is hepatotoxic. This patient has high serum alkaline phosphatase and low serum albumen, on the other hand, the biologic therapy is highly expensive and unaffordable by the patient.

\section{Conclusion}

In the absence of liver disease and alcohol consumption excluded by history, examination and investigations, high serum alkaline phosphatase is considered a relative contraindication to methotrexate therapy that can be given successfully and safely to patients with severe forms of psoriasis under close supervision in the ward.

\section{Recommendation}

Methotrexate is recommended for the treatment of severe morbid forms of psoriasis.

For those who can afford or in good centers the other option is the biologic therapy or cyclosporine mainly in patients with absolute contraindications to methotrexate.

\section{References}

[1] Rapp, S. R., Feldman, S. R., Exum, M. L., et al. (1999). Psoriasis causes as much disability as other major medical diseases. Journal of the American Academy of Dermatology, vol. 41, p. 401e7

[2] National Institute for Health and Clinical Excellence. (2008). Infliximab for the treatment of psoriasis. Retrieved from: http://www.nice.org.UK/TA134> (last accessed July 25, 2008).

[3] Alakloby, O. M. (2005). Pattern of skin disease in eastern Saudi Arabia. Saudi Medical Journal, vol. 26, p. 1610.

[4] National Institute for Health and Clinical Excellence. (2008). Adalimumab for the treatment of psoriasis. Retrieved from: http://www.nice.org.UK/TA146 (last accessed 
August 30, 2008).

[5] Roenigk, H. H., Auerbach, R., Maibach, H. I., et al. (1998). Methotrexate in psoriasis: consensus conference. Journal of the American Academy of Dermatology, vol. 38, pp. 478-485.

[6] Roenigk, H. H., Maibach, H. I., and Weinstein, G. P. (1973). Methotrexate therapy for psoriasis, guideline revisions. Archives of Dermatology, vol. 108, p. 35.

[7] Roenigk, H. H., Auerbach, R., Maibach, H. I., et al. (1982). Methotrexate guidelinesrevised. Journal of the American Academy of Dermatology, vol. 6, pp. 145-155.

[8] Martyn-Simmons, C. L., Rosenberg, W. M. C., Cross, R., et al. (2014). Validity of noninvasive markers of methotrexate-induced hepatotoxicity: a retrospective cohort. Dermatology, vol. 171, no. 2, pp. 267-273. Retrieved from: https://doi.org/10.1111/bjd. 12782

[9] Peckham, P. E., Weinstein, G. D., and McCullough, J. L. (1987). The treatment of severe psoriasis, a national survey. Archives of Dermatology, vol. 123, pp. 1303-1307.

[10] Dahlman-Ghozlan, K., Ortonne JP, Heilborn J, et al. Altered tissue expression pattern of cell adhesion molecules, ICAM-1, E-selectin and VCAM-1, in bullous pemphigoid during methotrexate therapy. Exp Dermatol 2004;13:65e9.

[11] Montaudi,_E. H., Sbidian, E., Paul, C., et al. (2011). Methotrexate in psoriasis: a systematic review of treatment modalities, incidence, risk factors and monitoring of liver toxicity. Journal of the European Academy of Dermatology and Venereology, vol. 25, p. 12 .

[12] Retrieved from: https://www.researchgate.net. 